\title{
A prospective evaluation of the impact of trans-rectal prostate biopsy on the voiding function of patients
}

\author{
Musliu Adetola Tolani* ${ }^{*}$, Muhammed Ahmed, Lovely Fidelis, Mudi Awaisu, Abdullahi Sudi, Oyelowo Nasir, \\ Ahmad Tijjani Lawal, Ahmad Bello and Hussaini Yusuf Maitama
}

\begin{abstract}
Background: Biopsy-related inflammatory edema, urethral blood clots and sphincteric spasm could lead to postbiopsy voiding dysfunction. This study examines the impact of trans-rectal prostate biopsy on the voiding function of patients in our institution.

Methods: Between February 2017 and July 2018, quasi-experimental study was carried out on 68 patients with indication(s) for prostate biopsy. Prior to the biopsy, IPSS (International Prostate Symptoms Score) and $Q_{\max }$ (peak flow rate) of each patient were determined. These were re-assessed 7 and 14 days after biopsy, and compared to baseline data. Analysis was done using Statistical Package for Social Science with $p<0.05$ considered significant.

Results: Post-biopsy acute urinary retention occurred in $4.4 \%$ of the patients. The total IPSS (12.5 vs. 14.7, $p=0.003)$, IPSS storage sub-score (6.7 vs. 8.3, $p=0.001)$, bother score (3.3 vs. 3.6, $p=0.025)$ and $Q_{\max }(18.7 \mathrm{vs} .15 .9 \mathrm{ml} / \mathrm{s}, p=0.001$ ) significantly deteriorated from the baseline value on the 7 th day post-biopsy. However, there was no significant difference in these variables in comparison with baseline by the 14th day after the biopsy. Patients with moderate IPSS and normal $Q_{\max }$ at baseline had significantly worsened values by the 7th post-biopsy day $(p=0.002$ and $p=0.001$, respectively) while those with lower baseline bother score had a higher tendency for worsening of their bother score.
\end{abstract}

Conclusions: Trans-rectal prostate biopsy causes significant worsening of lower urinary tract symptoms and associated deterioration in quality of life, which spontaneously resolved by the second week in the patients.

Keywords: Lower urinary tract symptoms, Prostate biopsy, Quality of life, Urinary retention, Voiding

\section{Background}

Prostate cancer constitutes a major public health burden worldwide. It is the second most common cancer in men following lung cancer which is the most common cancer in men globally [1]. The diagnosis of prostate cancer is hinged on histopathological analysis of the tissue following prostate biopsy or at post-mortem [2].

Just as the indications for prostate biopsy have evolved over the years, the protocols for the procedure have also

\footnotetext{
*Correspondence: adetolatolani@yahoo.com
}

Division of Urology, Department of Surgery, Ahmadu Bello University and Ahmadu Bello University Teaching Hospital, P.M.B. 06, Shika-Zaria, Kaduna State, Nigeria gone through several modifications. This is related to the quest for an increase in cancer detection rates and the need for reduction in complications associated with the procedure. Initially, sextant biopsy was the standard procedure, but presently, extended biopsy is increasingly being advocated for use in initial prostate biopsy to optimize cancer detection. The application of saturated biopsy is currently recommended for repeat biopsy following at least two negative results of extended biopsy in those with abnormal digital rectal examination findings, rising prostate specific antigen (PSA) or persistent atypical small acinar proliferation [3].

Complications could occur following prostate biopsy procedures. These can be broadly classified as infectious 
and non-infectious complications. Non-infectious complications include voiding dysfunction, bleeding, erectile dysfunction and vaso-vagal episodes [4]. Voiding dysfunction may present as worsening of voiding function or acute urinary retention. It could affect the quality of life of the patient after the procedure. Its duration is variable and depends on several factors.

This aim of this study was to examine the impact of trans-rectal prostate biopsy on the voiding of patients in our institution.

\section{Methods}

\subsection{Study population and selection criteria}

All adult male patients seen in our urology division between February 2017 and July 2018 with indications for prostate biopsy (suspicious findings on digital rectal examination, serum prostate specific antigen greater than $4.0 \mathrm{ng} / \mathrm{ml}$ or prostate specific antigen density greater than $0.15 \mathrm{ng} / \mathrm{ml} / \mathrm{g}$ ) who could void spontaneously as evidenced by peak urine flow measurements were considered for inclusion into this quasi-experimental study. Patients with acute urinary retention on indwelling suprapubic or urethral catheter, those having ongoing haematuria associated with clot passage, those with bleeding disorders, those on alpha-adrenergic receptor blockers or 5-alpha reductase inhibitors, and those with history of prior prostate surgery were excluded. Other exclusion criteria included the presence of Parkinson's disease, previous cerebrovascular accident or other neurological disease which could affect voiding function, and diabetes mellitus.

This study was approved by the Institutional Health Research Ethics Committee and informed consent was obtained from all patients recruited for the study.

\subsection{Study protocol}

Following patient enrollment, those on anticoagulants and acetyl salicylic acid were instructed to stop taking it at least 3-5 days and 7-10 days before the procedure, respectively, while those with ongoing urinary tract infection, as diagnosed by a positive urine culture, were treated with antibiotics according to the antimicrobial sensitivity pattern of the cultured organism. All patients on antibiotics for the treatment of infection were allowed to complete such treatment, and clearance of infection was determined clinically and confirmed by negative urine culture before their biopsies were taken.

Data on the patient's demographic, clinical and laboratory details were obtained and entered into the proforma. Baseline International Prostate Symptoms Score (IPSS) and bother scores were determined just before the procedure using IPSS questionnaire. This instrument has a set of 7 questions assessing three storage symptoms of frequency, urgency and nocturia, and four voiding symptoms of incomplete emptying, intermittency, weak stream and straining. These are scored from zero to five points with the total IPSS score ranging from 0 to 35 and its symptom severity graded as mild (0-7), moderate (8-19) and severe (20-35). The eighth question in this questionnaire, known as bother score which is scored from zero to six points, evaluates the disease-specific quality of life of the patient [5]. In the event that patients were not literate enough to complete the IPSS questionnaire, they were assisted to do so. Uroflowmetry was also done just before the prostate biopsy using the Ark Meditech Uroflowmeter, Urol 010 Model, to determine the peak flow rate (PFR).

A Mindray Digital Ultrasonic Diagnostic Imaging System, DP-20 Model with a $6.5 \mathrm{~Hz}$ end firing biplanar probe, was used for Trans-rectal Ultrasound Scan (TRUS) to determine the prostate volume. The maximal anteroposterior and transverse diameter were determined on transverse imaging, while the craniocaudal diameter was determined on sagittal imaging. The prostate volume was calculated using the ellipsoid formula $(\pi / 6 \times$ anteroposterior diameter $\times$ transverse diameter $\times$ craniocaudal diameter).

All patients received intravenous Gentamicin as antibiotic prophylaxis $30 \mathrm{~min}$ before the procedure. Patient was placed on the left lateral decubitus position. Ten milliliters of $2 \%$ intra-rectal Lidocaine gel was instilled into the rectum. Five minutes after application of the anesthesia, an 18G Tru-cut biopsy needle mounted on a semiautomated biopsy gun (Bard ${ }^{\circledR}$ Magnum ${ }^{\circledR}$ Reusable Core Biopsy System) was inserted into the rectum to carry out a systematic sextant prostate biopsy lateral to the midgland. The whole procedure was performed by the same investigator using the same technique in all the patients.

IPSS, bother score and PFR were re-assessed in comparison with the baseline values, 7 and 14 days after the biopsy. Patients who developed post-biopsy acute urinary retention were also noted, and bladder decompression was carried out in them using an indwelling urethral catheter. Histological results of the patients were obtained in the second week after the biopsy. Patients were given medical therapy for lower urinary tract symptoms when negative tissue histology for malignancy was confirmed after they exited the two-week study window.

\subsection{Outcome measures}

The outcome measures for voiding function in this study were the development of acute urinary retention and a difference between PFR values or a variation in IPSS values at specific time points while a change in the bother score was used as a measure of alteration in the 
Lower Urinary Tract Symptoms-related quality of life in the patients.

\subsection{Statistical analysis}

G*Power Version 3.1.9.2 was used for power analysis (Franz F., Universität Keil, Germany), while data analysis was done using Statistical Package for Social Sciences (SPSS) software version 20.0 (IBM Corp., Armonk, New York, USA). The data were summarized as mean and standard deviation for continuous variables, and frequency and percentage for categorical variables. Paired samples $t$ test was used to compare pre- and post-test data while Spearman's and Pearson's correlation test for the determination of the relationship between two variables. Independent samples t-test was used to compare peak flow rate between two groups. The $p$ value was considered to be significant if $<0.05$.

Table 1 Baseline characteristics of the study population

\begin{tabular}{lcccc}
\hline Variables & Mean & $\begin{array}{c}\text { Standard } \\
\text { deviation }\end{array}$ & Minimum & Maximum \\
\hline Age (years) & 64.9 & 8.4 & 41 & 85 \\
Prostate volume (g) & 60.5 & 49.4 & 8 & 277.1 \\
PSA (ng/ml) & 28.5 & 31.6 & 4 & 116.6 \\
IPSS-T & 12.8 & 7.3 & 2 & 29 \\
IPSS-S & 6.7 & 4.0 & 1 & 17 \\
IPSS-V & 6.2 & 4.8 & 0 & 17 \\
IPSS-V/S & 1.2 & 1.4 & 0 & 8 \\
Bother Score & 3.3 & 1.6 & 0 & 6 \\
PFR (ml/s) & 18.3 & 10.7 & 6 & 62 \\
\hline
\end{tabular}

IPSS: International Prostate Symptom Score, IPSS-T: total IPSS score, IPSS-S: IPSS storage symptoms sub-score, IPSS-V: IPSS voiding symptoms sub-score, IPSSV/S: IPSS voiding to storage symptoms ratio, PFR: peak flow rate, PSA: prostate specific antigen

\section{Results}

A total of 81 patients who met the inclusion criteria were enrolled for the study from February 2017 to July 2018. Thirteen of them were lost to follow-up. A total of 68 patients' data were finally analyzed and this had a power of 0.982 in detecting statistical significance.

The mean age of the study population was 64.9 years while the median prostate volume and PSA were $47.8 \mathrm{~g}$ and $15.4 \mathrm{ng} / \mathrm{ml}$, respectively. Other clinical characteristics are shown in Table 1. Within the IPSS groups, the proportion of patients with mild, moderate and severe IPSS at baseline were 16 (23.5\%), 37 (54.4\%) and 15 (22.1\%), respectively, while their baseline IPSS voiding to storage symptoms ratio were $0.5 \pm 0.8,1.5 \pm 1.7$ and $1.2 \pm 0.5$, respectively.

Acute urinary retention (AUR) occurred in $4.4 \%$ of the patients within the first $24 \mathrm{~h}$ after biopsy. In comparison with baseline, there was significant worsening of total IPSS, IPSS storage symptoms sub-score, bother score and PFR on the 7th day post-biopsy but no significant difference between the baseline and 14th day post-biopsy values (Table 2).

Patients with moderate IPSS and those with PFR $\geq 15 \mathrm{ml} / \mathrm{s}$ had significant change in IPSS and PFR values, respectively, from baseline by the 1 st week after the biopsy $(p=0.002$ and $p=0.001$, respectively) (Table 3 ). There was a significant but weak positive correlation between change in PFR and change in total IPSS one week after the biopsy $(p=0.032$, $r=0.266$ ). There was also a significant negative correlation between the baseline bother score and the bother score change ( $p=0.004, r=-0.354)$ (Fig. 1). However, the change in baseline and 7th day post-biopsy PFR in those who voided did not differ significantly between those with negative and positive histology for prostate cancer $(8.0 \pm 3.6 \mathrm{ml} / \mathrm{s}$ versus $7.9 \pm 3.6 \mathrm{ml} / \mathrm{s}, p=0.930)$.

Table 2 Comparison of the baseline and post-biopsy IPSS, bother score and PFR in patients who voided after prostate biopsy

\begin{tabular}{|c|c|c|c|c|c|c|}
\hline \multirow[t]{2}{*}{ Variables } & \multicolumn{3}{|c|}{ Timing in relation to prostate biopsy } & \multicolumn{3}{|l|}{$p$ values* } \\
\hline & Do & D7 & D14 & D0 versus D7 & D7 versus D14 & D7 versus D14 \\
\hline IPSS-T & $12.5 \pm 7.2$ & $14.7 \pm 8.2$ & $11.3 \pm 8.1$ & 0.003 & 0.001 & 0.138 \\
\hline IPSS-S & $6.7 \pm 3.9$ & $8.3 \pm 5.0$ & $6.3 \pm 4.3$ & 0.001 & 0.001 & 0.446 \\
\hline IPSS-V & $5.9 \pm 4.8$ & $6.1 \pm 4.9$ & $5.0 \pm 4.6$ & 0.701 & 0.053 & 0.082 \\
\hline Bother Score & $3.3 \pm 1.6$ & $3.6 \pm 1.7$ & $2.9 \pm 1.7$ & 0.025 & 0.001 & 0.072 \\
\hline PFR (ml/s) & $18.7 \pm 10.8$ & $15.9 \pm 10.4$ & $17.7 \pm 10.5$ & 0.001 & 0.039 & 0.294 \\
\hline
\end{tabular}

All variables are expressed as mean \pm standard deviation

IPSS: International Prostate Symptom Score, IPSS-T: total IPSS score, IPSS-S: IPSS storage symptoms sub-score, IPSS-V: IPSS voiding symptoms sub-score, PFR: peak flow rate, D0: baseline values, D7: 7th day post-biopsy values, D14: 14th day post-biopsy values

${ }^{*} p$ value (paired samples $t$ test) $<0.05$ is significant 
Table 3 Comparison of the baseline and 7th day postbiopsy total IPSS and PFR in patients with different baseline IPSS and PFR categories who voided after prostate biopsy

\begin{tabular}{lccc}
\hline Categories & Baseline & 7th day post-biopsy & $\boldsymbol{p}$ value* \\
\hline IPSS categories & & & \\
Mild (0-7) & $3.9 \pm 2.3$ & $5.8 \pm 4.1$ & 0.125 \\
Moderate (8-19) & $12.1 \pm 2.8$ & $15.3 \pm 6.0$ & 0.002 \\
Severe (20-35) & $23.5 \pm 2.9$ & $23.4 \pm 6.0$ & 0.933 \\
PFR categories & & & \\
Normal ( $\geq 15 \mathrm{ml} / \mathrm{s})$ & $26.3 \pm 10.5$ & $21.6 \pm 9.9$ & 0.001 \\
Equivocal (10-14 ml/s) & $12.7 \pm 1.2$ & $13.5 \pm 9.5$ & 0.834 \\
Obstructed (<10 ml/s) & $8.0 \pm 0.6$ & $8.2 \pm 7.6$ & 0.939 \\
\hline
\end{tabular}

All variables are expressed as mean \pm standard deviation

IPSS: International Prostate Symptom Score, PFR: peak flow rate

${ }^{*} p$ value (paired samples $t$ test) $<0.05$ is significant

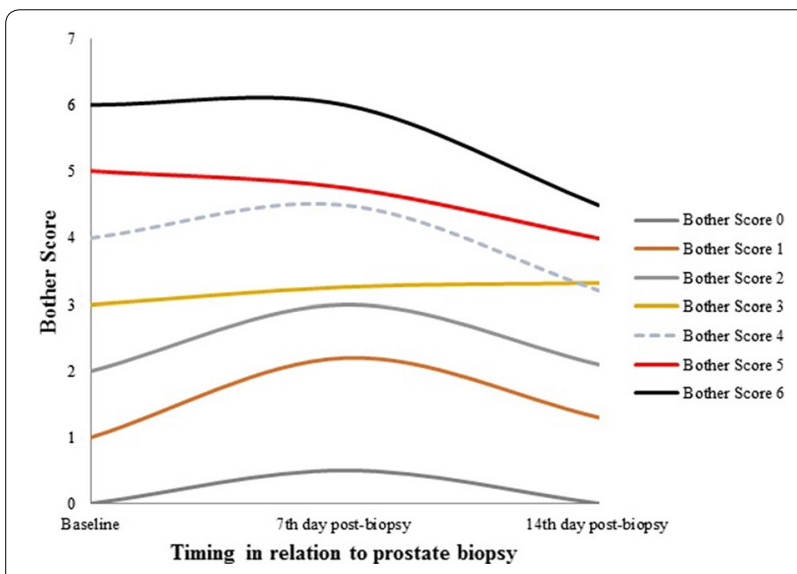

Fig. 1 Line diagram showing trends in the 7th and 14th day average bother scores at different baseline bother scores in patients who voided after prostate biopsy

\section{Discussion}

In this study, it was observed that $4.4 \%$ of the patients developed AUR within a period of $24 \mathrm{~h}$ after the biopsy. Mbaeri et al. [6] reported that AUR occurred in 5\% of their patients. The similarity in these figures could be related to the use of sextant biopsy protocol in both studies. However, Aktas et al. [7] reported a slightly higher incidence of $6.5 \%$ in their study, where 10 biopsy cores were taken. It is possible that the higher incidence of instrumentation-related trauma induced by the increased number of cores during the biopsy procedure could account for the disparity in the incidence rates. To further support this assertion, Kum et al. [8] reported a postbiopsy AUR incidence rate of $12.8 \%$ in their $32-35$ core biopsy, which is over three times the rate observed in this study. The marked disparity in these values could be due to the lower mean age (64.9 years) in our study compared to the result (66.2 years) of their study. It could also be as a result of the lower median prostate volume $(47.8 \mathrm{~g})$ in patients enrolled in this study in contrast to the relatively larger prostate volume $(59.6 \mathrm{~g})$ in their patients as there is a potential for greater reactive edema and subsequent obstruction in larger prostates.

Findings in this study which showed a significant increase in the average total IPSS and IPSS storage symptoms sub-score values on the 7th post-biopsy day demonstrate that patients experience worsening of lower urinary tract symptoms, particularly irritative symptoms, within one week of biopsy. This is in consonance with the observation of Bozlu et al. who reported a significantly elevated mean total IPSS on the 7th day after their 12-core biopsy procedure and Quaresima et al. who noted that patients reported irritative urinary symptoms following their 12-16 core prostate biopsy $[9,10]$. In contrast to the outcome of this study, Chung et al. [11], who performed octant biopsy, reported that there was no significant difference between the baseline and 7th day postbiopsy mean total IPSS values. This could be because the baseline mean prostate volume in their patients (40 g) was smaller than the average prostate volume reported in the present study $(47.8 \mathrm{~g})$. Larger prostates are more prone to vigorous biopsy-related trauma during maneuvers to reach the base of the prostate [7]. Another reason for this variance might be that the decrease in urine flow rate which their patients experienced after the biopsy $(1.6 \mathrm{ml} / \mathrm{s})$ was comparatively lower than the decrease in flow rate patients experienced in the present study $(2.8 \mathrm{ml} / \mathrm{s})$, and thus, their flow rate decrease might not be significant enough to cause symptom perception.

The observation that the 14th day post-biopsy mean total IPSS value significantly reduced in comparison with the 7th day post-biopsy mean total IPSS value implies that there was a significant improvement in voiding by the second week after the biopsy. Unlike this research which was designed to determine whether improvement in voiding impairment starts by the second week after the procedure, most researchers reported IPSS findings in their patients 7 and 30 days after prostate biopsy. Bozlu et al. noted that there was a 2.6 point reduction in total IPSS values between the 7th and 30th day after prostate biopsy [9]. They also observed that there was no significant difference between the baseline and 30th day postbiopsy IPSS values in their patients (15.2 versus 15.6). This implies that by the 30th day after the biopsy, symptoms reverted back to pre-biopsy level in their patients. The findings of this study thus re-enforce the assertion of Bozlu et al. [9] that worsening of IPSS is transient in the patients. 
The result of this research also revealed that the patient sub-group with moderate IPSS values (8-19) at baseline had a significant increase in mean total IPSS scores 7 days after the biopsy, while those with mild or severe symptoms did not experience a significant change in their symptom score. Voiding dysfunction is defined by an IPSS voiding to storage symptoms ratio of greater than 1.0 [12]. Thus, our finding could be related to the fact that the highest IPSS voiding to storage symptoms ratio (1.5) was recorded among patients with moderate baseline IPSS. Temi and Gbenga [13] also observed that the proportion of patients with moderate symptoms increased from $40.5 \%$ at baseline to $51.9 \% 7$ days after their six-core prostate biopsy. In addition, Klein et al. [14] observed an increase in the proportion of patients with moderate symptoms from $31.8 \%$ to $38.6 \%$ one week after their 10 -core prostate biopsy. Thus, baseline total IPSS values may be a predictor of the severity of symptoms of postbiopsy voiding impairment and could be taken into cognizance during the pre-procedural counselling of patients about the risk of this complication.

Based on the use of peak flow rate as an objective measure of voiding function, this study revealed that prostate biopsy leads to voiding impairment because there was a significant decrease in the mean PFR one week after the biopsy. This finding agrees with the result of Chung et al. [11] who observed a similar significant decrease in the PFR on the 7th post-biopsy day and also showed a strong negative correlation between baseline PFR and PFR change. The present study showed that only patients with baseline peak flow rate $15 \mathrm{ml} / \mathrm{s}$ and above had a significant decrease in their mean PFR one week after the biopsy. This flow rate value is traditionally used for patients who are not obstructed [15]. Thus, patients who are unobstructed at baseline are likely to have worsening of their urine flow rate after prostate biopsy. However, decrease in PFR does not always translate to the worsening of patients' symptoms as there was a weak positive correlation between the change in PFR and total IPSS one week after the biopsy. There was, however, a significant increase in peak flow rate 14 days after the prostate biopsy. Aktas et al. [7] noted that prostate edema occurs as a result of trauma following prostate biopsy. The gradual resolution of this inflammatory edema could explain improvement in voiding function by the second week after the procedure.

There was a significant deterioration in the quality of life (QoL) of the patients one week after the prostate biopsy. This is in consonance with the research of Bozlu et al. [9], though the difference between the mean baseline and mean 7th day post-biopsy bother scores reported in their study was not significant. The lack of statistical significance may be due to the relatively smaller sample size (33 patients) in the control group of their study.

Patients with a lower baseline bother score had a higher tendency to have a worse deterioration in their quality of life than those with a higher baseline bother score. This may be because those with a lower baseline bother score are more sensitive and, thus, readily perceive minimal deterioration in their voiding function, leading to sudden alteration in their quality of life.

Alpha-adrenergic receptor blockers have a high affinity for the smooth muscle of the prostate and affect the relaxation of the prostate and bladder neck [16]. Chung et al. [11] reported a significant improvement in maximal flow rate from a pre-biopsy level of $12.1 \mathrm{ml} / \mathrm{s}$ to a value of $13.7 \mathrm{ml} / \mathrm{s}$ seven days post-biopsy in patients who had $0.2 \mathrm{mg}$ prophylactic dose of Tamsulosin for one week after the procedure. Sefik et al. [16] in their prospective randomized research also observed that patients on $0.4 \mathrm{mg}$ prophylactic dose of Tamsulosin experienced a significant increase in their peak flow rate $(0.8 \mathrm{ml} / \mathrm{s})$ compared to the decrease experienced by those that were not on it $(-1.7 \mathrm{ml} / \mathrm{s})$. Therefore, alpha-adrenergic receptor blockers could be used to minimize the complication of voiding dysfunction in patients undergoing prostate biopsy.

The lack of usage of pressure-flow studies to further define the voiding function of patients is a limitation of this study. Despite this, voiding was objectively assessed with peak urine flow rate measurements. The further analysis of peri-biopsy changes in IPSS voiding and storage symptoms sub-scores and the investigation of the earliest week of onset of resolution of post-biopsy voiding impairment form the strength of this study.

\section{Conclusions}

This study has shown that there may be significant Lower Urinary Tract Symptoms and associated alteration in the quality of life, which spontaneously resolves by the second week after prostate biopsy. Pre-prostate biopsy evaluation of patients should include PFR, IPSS, and QoL to assess baseline voiding parameters in order to counsel patients on the risk of this complication as well as guide the peri-biopsy need for prophylactic alpha-adrenergic receptor blockers.

\section{Acknowledgements \\ Not applicable.}

Authors' contributions

All authors were involved in the development of the project. MAT, LF, MAW and AS were involved in data management. MAT, ON and ATL did the data analysis and manuscript writing. MAT, MAH, AB and HYM carried out manuscript editing. All authors read and approved the final manuscript. 


\section{Funding}

This study received no external funding.

\section{Availability of data and materials}

The datasets used and/or analyzed during the current study are available from the corresponding author on reasonable request.

\section{Ethics approval and consent to participate}

Ethical approval was obtained from the Health Research Ethics Committee of Ahmadu Bello University Teaching Hospital, Shika-Zaria, Nigeria (ABUTH/ HREC/X9/2016), and written informed consent was obtained. Participation was voluntary and confidentiality was maintained throughout the study.

\section{Consent for publication}

Not applicable.

\section{Competing interests}

The authors declare that they have no competing interests.

Received: 1 April 2020 Accepted: 10 August 2020

Published online: 02 November 2020

\section{References}

1. Adeloye D, David R, Aderemi A, Iseolorunkanmi A, Oyedokun A, Iweala E et al (2016) An estimate of the incidence of prostate cancer in Africa: a systematic review and meta-analysis. PLoS ONE 11:1-18

2. Roberts RO, Bergstralh EJ, Peterson NR, Bostwick DG, Lieber MM, Jacobsen SJ (2000) Positive and negative biopsies in the pre-prostate specific antigen and prostate specific antigen eras, 1980 to 1997. J Urol 163:1471-1475

3. El-Hakim A (2010) CUA guidelines on prostate biopsy methodology. Can Urol Assoc J 4:89-94

4. Loeb S, Vellekoop A, Ahmed HU, Catto J, Emberton M, Nam R et al (2013) Systematic review of complications of prostate biopsy. Eur Urol 64:876-892

5. Sanman KN, Shetty R, Adapala RR, Patil S, Prabhu GGL, Venugopal P (2020) Can new, improvised Visual Prostate Symptom Score replace the International Prostate Symptom Score? Indian perspective. Indian J Urol 36:123-129

6. Mbaeri TU, Abiahu JA, Orakwe JC, Oranusi CK, Nwofor AME, Obiesie EA et al (2017) Complications of prostate biopsy in two centres in Anambra State. Orient J Med 29:133-139
7. Aktas BK, Bulut S, Gokkaya CS, Ozden C, Salar R, Aslan Y et al (2014) Association of prostate volume with voiding impairment and deterioration in quality of life after prostate biopsy. Urology 83:617-621

8. Kum F, Jones A, Nigam R (2018) Factors influencing urinary retention after transperineal template biopsy of the prostate: outcomes from a regional cancer centre. World J Urol 36:1-6

9. Bozlu M, Ulusoy E, Doruk E, Cayan S, Canpolat B, Schellhammer PF et al (2003) Voiding impairment after prostate biopsy: does tamsulosin treatment before biopsy decrease this morbidity? Urology 62:1050-1053

10. Quaresima L, Lacetera V, Leone L, Montesi L, Cantoro U, Polito M et al (2014) The impact of repeated prostate biopsies on sexual function and urinary symptoms in patients with diagnosis of Atypical Small Acinar Proliferation (ASAP): Can ecodoppler reduce side effects? Arch Ital Urol Androl 86:356-358

11. Chung SJ, Jung SI, Ryu JW, Hwang EC, Kwon DD, Park K et al (2015) The preventive effect of tamsulosin on voiding dysfunction after prostate biopsy: a prospective, open-label, observational study. Int Urol Nephrol 47:711-715

12. Jiang $\mathrm{YH}$, Liao CH, Kuo HC (2018) Role of bladder dysfunction in men with lower urinary tract symptoms refractory to alpha-blocker therapy: a video-urodynamic analysis. LUTS 10:32-37

13. Temi AP, Gbenga OJ (2017) Prevalence of voiding dysfunction after finger-guided prostate needle biopsy in Nigerian men. Med Surg Uro $6: 1-4$

14. Klein T, Palisaar RJ, Holz A, Brock M, Noldus J, Hinkel A (2010) The impact of prostate biopsy and periprostatic nerve block on erectile and voiding function: a prospective study. J Urol 184:1447-1452

15. Oranusi C, Nwofor A, Mbonu O (2017) Correlation between international prostate symptom score and uroflowmetry in patients with benign prostatic hyperplasia. Niger J Clin Pract 20:454-458

16. Sefik E, Eker A, Gunlusoy B, Celik S, Bozkurt IH, Basmaci I et al (2020) The effect of alpha blocker treatment prior to prostate biopsy on voiding functions, pain scores and health-related quality-of-life outcomes: a prospective randomized trial. Prog Urol 30:198-204

\section{Publisher's Note}

Springer Nature remains neutral with regard to jurisdictional claims in published maps and institutional affiliations.

\section{Submit your manuscript to a SpringerOpen ${ }^{\circ}$ journal and benefit from:}

- Convenient online submission

- Rigorous peer review

- Open access: articles freely available online

- High visibility within the field

Retaining the copyright to your article

Submit your next manuscript at $\boldsymbol{\nabla}$ springeropen.com 